\title{
A Study of State Estimation Algorithms in an OktoKopter
}

\author{
S. A. Quadri, Othman Sidek and Azizul bin Abdullah \\ Collaborative Microelectronic Design Excellence Centre (CEDEC) \\ Universiti Sains Malaysia, Engineering Campus, Nibong Tebal \\ Malaysia, 14300 \\ reachquadri@yahoo.com
}

\begin{abstract}
The prospective applications of unmanned aerial vehicle (UAV) have marked its significance in various military and non-military applications. On-board sensor accuracy and state estimation algorithms are important issues related with the performance aspects. Our study is focused on OktoKopter, which is one of the successful universal aerial platforms. The multi-rotor aircraft is equipped with global positioning system (GPS), compass, altitude control and telemetry, etc., thus the features make it powerful and extremely versatile. In this paper, we first present a sensor fusion model, and then proceed with comparison of three state estimation algorithms, namely Kalman, extended Kalman filter (EKF) and unscented Kalman filter(UKF).The performance of UKF is found to be the best; the result being tallied with theoretical notion of the algorithm with practical experimented data.
\end{abstract}

Keywords: State estimation, Algorithms, Kalman filter, Extended Kalman filter, Unscented Kalman filter, MikroKopter, OktoKopter, Estimation error, Matlab

\section{Introduction}

MikroKopter are electrically driven, universal flying platforms that are ideal for capturing aerial photos and videos. The electronic stabilization makes the controlling and steering very easy. MikroKopter are equipped with GPS and can automatically hold position and altitude and even fly to GPS coordinates locations automatically. The most popular MikroKopter, namely OktoKopter was commercially purchased by our research centre [1]. The kit comes with over 400 discrete pieces that must be first screwed, bolted, and soldered together. Step by step instructions for assembling the parts were followed as given in the instruction manual [2].

Every man-made object in the space such as space stations, spacecraft and satellites are susceptible to many disturbances in space environment that creates an undesirable translation and rotational motions of the spacecraft or satellites. Estimation in nonlinear systems is extremely important because almost all practical systems from target tracking [3] to vehicle navigation, from chemical process plant control [4] to dialysis machines involve nonlinearities of one kind or another.

The problem of state estimation concerns the task of estimating the state of a process while only having access to noisy and/or inaccurate measurements from that process. It is a ubiquitous problem setting, encountered in almost every discipline within science and engineering. Thus, selection of proper estimation algorithm plays a vital role in flight dynamics domain. 
In our project, we first utilize the notion of sensor fusion, which obviously increases the system performance, and then we try to compare three estimation algorithms. Experimental data from the OktoKopter are obtained and simulations are carried over Matlab platform.

The paper is organized as follows, Section 2 concisely outlines the terms and technical specifications of the aircraft. Section 3 proposes a sensor fusion model to fuse the data. Section 4 discuss the theoretical background of state estimation algorithms, Section 5 deals with the results and analysis, thus suggesting UKF to be better choice than KF and EKF.

\section{Materials and Methods}

All In this section, we discuss the technical details of the aircraft, the basic terms and the flight control mechanism.

\subsection{OktoKopter}

MikroKopters are easy to use and highly reliable, that has increased its versatility. Equipped with GPS, compass, altitude control, telemetry, etc., the MikroKopter can automatically hold the current altitude \& position, fly back automatically to the start position, show telemetry data such as power consumption, flight time, altitude and low voltage warning [2].

One of the most successful MikroKopter model is 'OktoKopter XL', the same commercially available in the market is purchased for the research and experimentation. It can carry cameras up to $2.5 \mathrm{~kg}$ and remain in the air about 20-30 minutes depending on the payload and the battery capacity. All functions of the OktoKopter can be controlled from the transmitter. This also provides the telemetry flight data on a display and by voice output. In addition, the camera can be controlled from the transmitter (photo or video release and even provides zoom functions). The live image from the camera is transmitted over a wireless connection to a monitor. Therefore, the pilot always sees the current image. Compared to a QuadroKopter with four motors, an OktoKopter with eight motors has extra redundancy and can carry more payloads. When a motor would fail on an OktoKopter, it can still be flown and safely landed.

The basic aircraft system comprises of printed circuit board (PCB) controller unit, protective hood, propellers, eight wings and wireless remote controller, the complete assembled set is shown in Figure 1. All the circuitry embedded on PCB is shielded by protected hood; the same is depicted in Figure 2. The acronym XL stands for heXa brushLess motor controller board, given by the manufacturer. A propeller is a type of fan that transmits power by converting rotational motion into the thrust. Propeller is one of the propulsion systems. The purpose of the propeller is to move the aircraft through the air. The propeller consists of two or more blades connected together by a hub. ARF (Almost ready to Fly) wireless transmitter system with different modes and buttons is shown in Figure 3. 


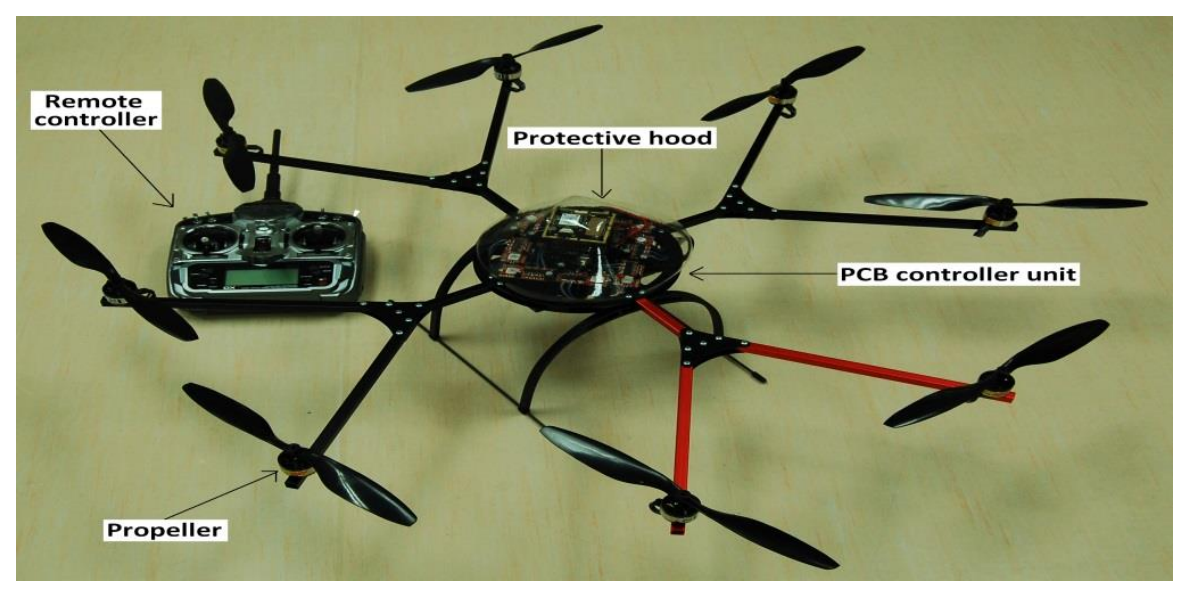

Figure 1. ARF-MikroKopter OktoXL

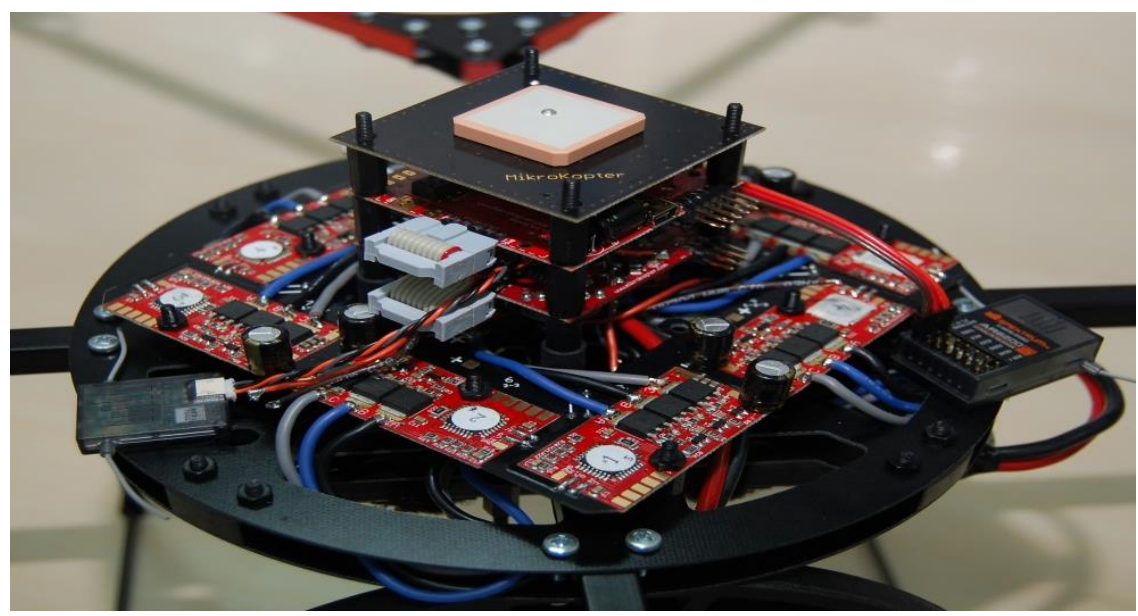

Figure 2. XL- Power Board

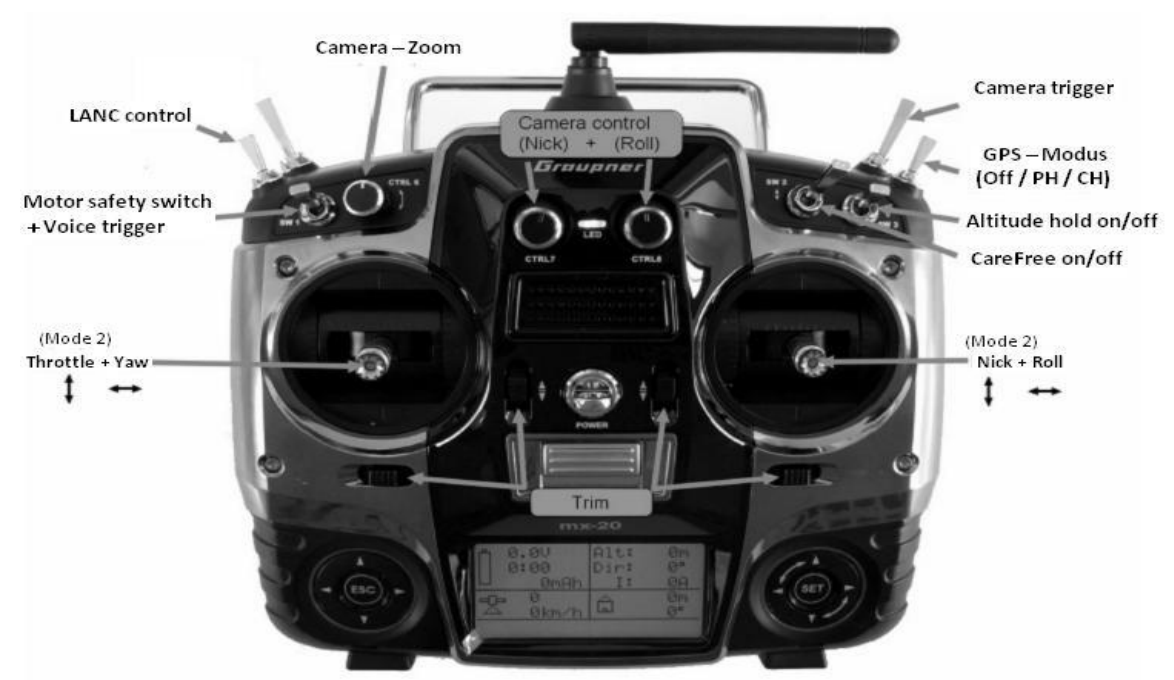

Figure 3. ARF Transmitter System 


\subsection{Basic Terms and Technical Specifications}

An inertial measurement unit works by detecting the current rate of acceleration using one or more accelerometers, and detects changes in the rotational attributes like pitch, roll and yaw using one or more gyroscopes. A magnetometer is also included in order to reduce the orientation drift. Roll, pitch and yaw are the three angles of rotation in three dimensions(X, Y and Z-axis) about the vehicle's center of mass. The three critical flight dynamics parameters are shown in Figure 4.

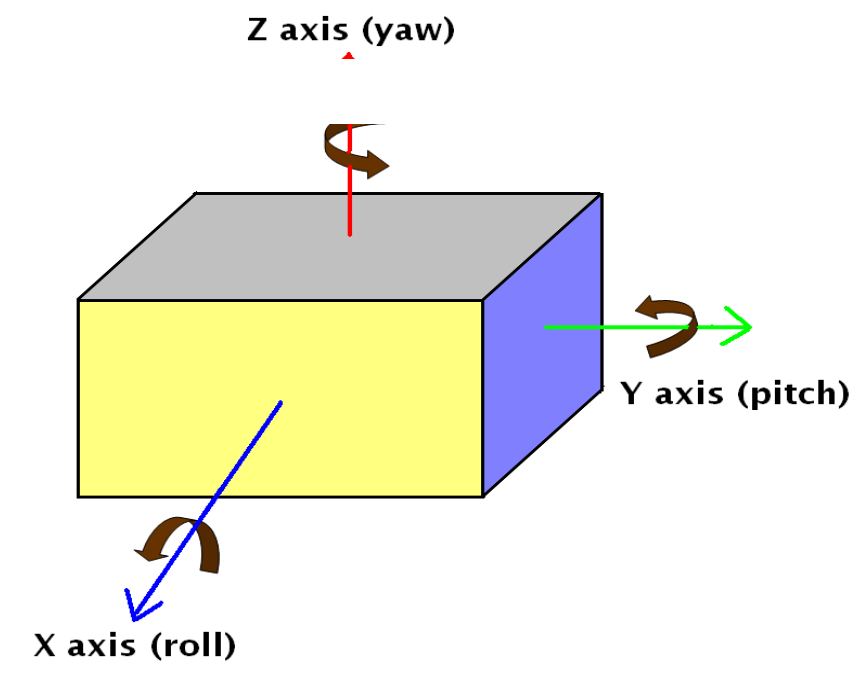

Figure 4. Three Angles of Rotation (yaw, pitch and roll)

By utilizing the accelerometer output, rotation around the $\mathrm{X}$-axis (roll) and around the $\mathrm{Y}$ axis (pitch) can be calculated. If Accel_X, Accel_Y, and Accel_Z are accelerometer measurements in the X-, Y- and Z-axes respectively, Equations 1 and 2 presents how the pitch and roll angles are calculated [5].

$$
\begin{array}{ll}
\text { Pitch }=\arctan \left(\frac{\text { Accel_X }}{(\text { Accel_X })^{2}+(\text { Accel_Z })^{2}}\right) & \text { Equation (1) } \\
\text { Roll }=\arctan \left(\frac{\text { Accel_Y }}{(\text { Accel_Y })^{2}+(\text { Accel_Z })^{2}}\right) & \text { Equation (2) }
\end{array}
$$

The MK-Flight Control board has five separate sensors that work together to maintain stable and controlled flight. Three Gyroscopes are installed on the board to sense angular movement in the three different geometric axis planes, an accelerometer capable of detecting movement speed in all three directions, and an atmospheric pressure sensor used for altitude control. Technical description of the aircraft is given in the Table 1. 
Table 1. Technical Specification of the Aircraft

\begin{tabular}{|l|l|}
\hline Model & ARF-MikroKopter OktoXL \\
\hline Drive & Electro drive \\
\hline Voltage / Current consumption: & $14,8 \mathrm{~V} /$ max. 100A \\
\hline Motors & Individually controlled Brushless 8 Motors \\
\hline Control & $\begin{array}{l}\text { Control via a suitable radio control (RC)-transmitter \& receiver } \\
\text { with Pulse-position modulation (PPM) Sum signal. }\end{array}$ \\
\hline Total weight & ARF-MikroKopter (without battery/payload) $=2050 \mathrm{~g}$ \\
\hline Battery brand and weight & LiPo (Lithium-Polymer-Accumulator battery ) with weight 520g \\
\hline Recommended maximum payload & $2500 \mathrm{~g}$ \\
\hline Allowable maximum total weight & $5000 \mathrm{~g}$ \\
\hline Size & $73 \times 73 \times 36(\mathrm{~W}$ x L $\mathrm{x}$ H) \\
\hline Flight times & $8-41$ minutes (Depending on the payload \& battery choice) \\
\hline Flight radius & $4 \mathrm{~km}$ \\
\hline Altitude & Up to $3000 \mathrm{~m}$ over sea level \\
\hline Temperature range & $-5^{\circ} \mathrm{C}$ to $+35^{\circ} \mathrm{C}(+23 \mathrm{~F}$ to $+95 \mathrm{~F})$ \\
\hline
\end{tabular}

Few basic abbreviations are also listed below which are often used during the operation.

- Gyroscope/Gyro - Electromechanical devices that measure the angular velocity of an object in degrees per second.

- Accelerometer (ACC) - A special gyro that determines the acceleration by measuring the force on a known sample mass.

- Center of Gravity (CG) - The average location of the weight of an object. The point at which the MK would balance if it were possible to suspend it at that point.

- MK - Abbreviation for Mikrokopter.

- MK Flight Control - Circuit board that contains all of the electronics needed for MK flight.

- Nick - MK pitch controlled by the right control stick on the radio transmitter in a forward/reverse direction.

- Roll - MK roll controlled by the right control stick on the radio transmitter in a right/left direction.

- YAW - MK rotation in a horizontal plane controlled by the left stick on the radio transmitter in a right/left direction.

- MEMS - Microelectromechanical systems is the technology of very small mechanical devices driven by electricity.

- EEPROM - Electrically Erasable Programmable Read-Only Memory and is a type of non-volatile memory used in computers and other electronic devices to store small amounts of data that must be saved when power is removed, e.g., calibration tables or device configuration. 
- Weight and Balance - The science of properly distributing load to operate the MK within proper weight limits and center of gravity location. Flight performance is greatly affected by the weight and balance.

\subsection{Flight Control Mechanism}

The flight control computer (FCC) is the most important and expensive board on the Mikrokopter. It contains the main processor, voltage transformers, and all the sensors that are necessary for the stable flight. The sensors that are mounted on the flight control board (FCB) are sophisticated electronic instruments that indicate the motion, altitude and acceleration of the MK while it is in flight mode. Without these sensors, the Mikrokopter would not be able to maintain level attitude, altitude, or follow the flight control inputs from the pilot.

The brain of the MK is the flight controller computer chip that contains the software to operate the MK. The controller uses the inputs from the sensors to calculate the MK attitude and acceleration that in turn controls the speed for each individual propeller and compensation needed for the stable flight. If the flight controller software is properly programmed and the sensors correctly calibrate, the Mikrokopter will remain steady in the air and respond smoothly to the flight commands from the pilot. While there are other systems on the MK that also need to be correctly set and operated, the proper understanding and setup of the flight control sensors is critical to quality flight characteristics.

The flight control firmware for the OktoKopter in our project is MikroKopter-Tool V1.80b. Interactive GUI interface of the software is shown in the Figure 5.

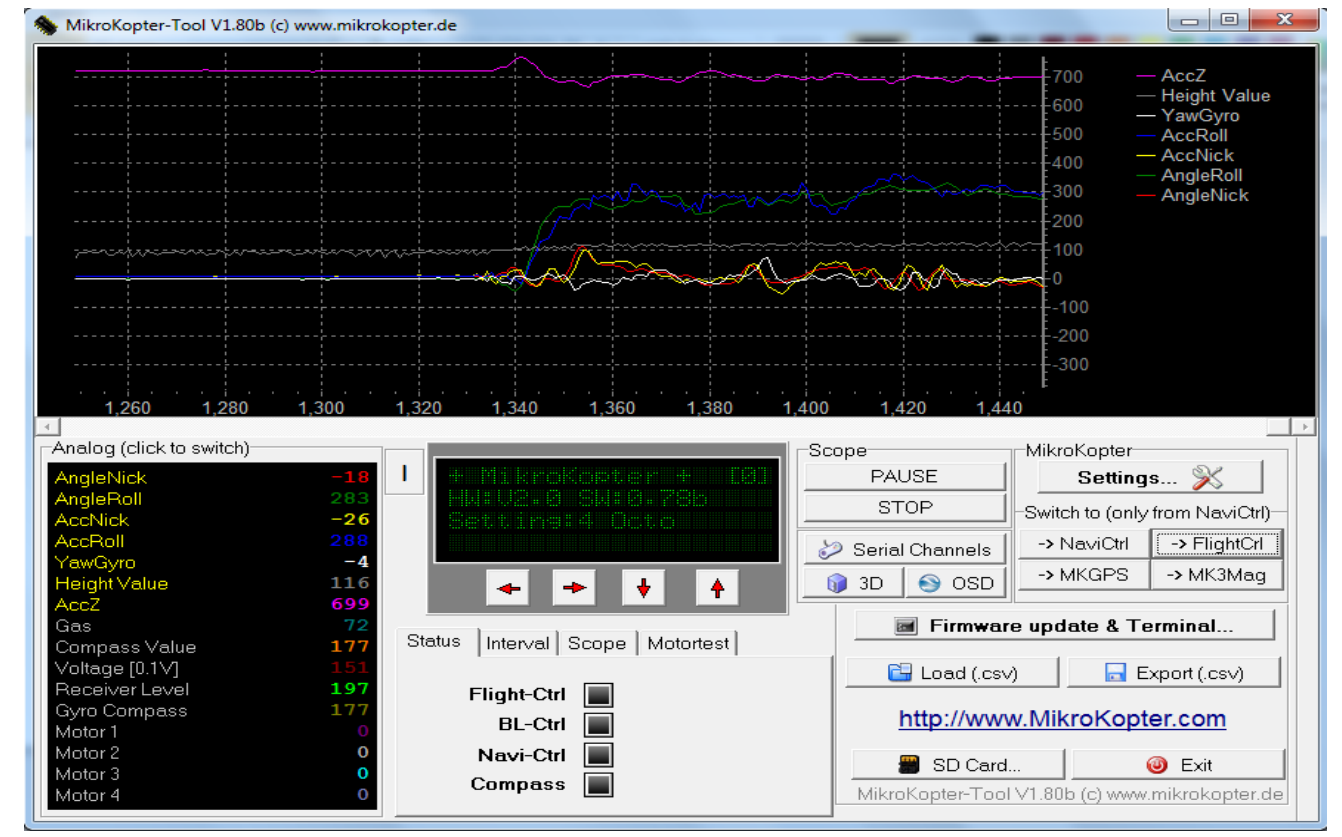

Figure 5. Screen shot of MK Flight Control Software

\section{Sensor Fusion Model}

The Data fusion techniques can combine data from multiple information sources to achieve improved accuracies and more specific inferences than by the use of a single source alone [6]. Sensor fusion has prominent role in improving performance and reducing estimation errors in inertial measurement units (IMU). [7-9]. 
The applied sensor fusion model is depicted in Figure 6. Flight control firmware (MikroKopter-Tool V1.80b) facilitates to obtain raw data from sensors in comma-separated values (CSV) to carry out Matlab simulations.

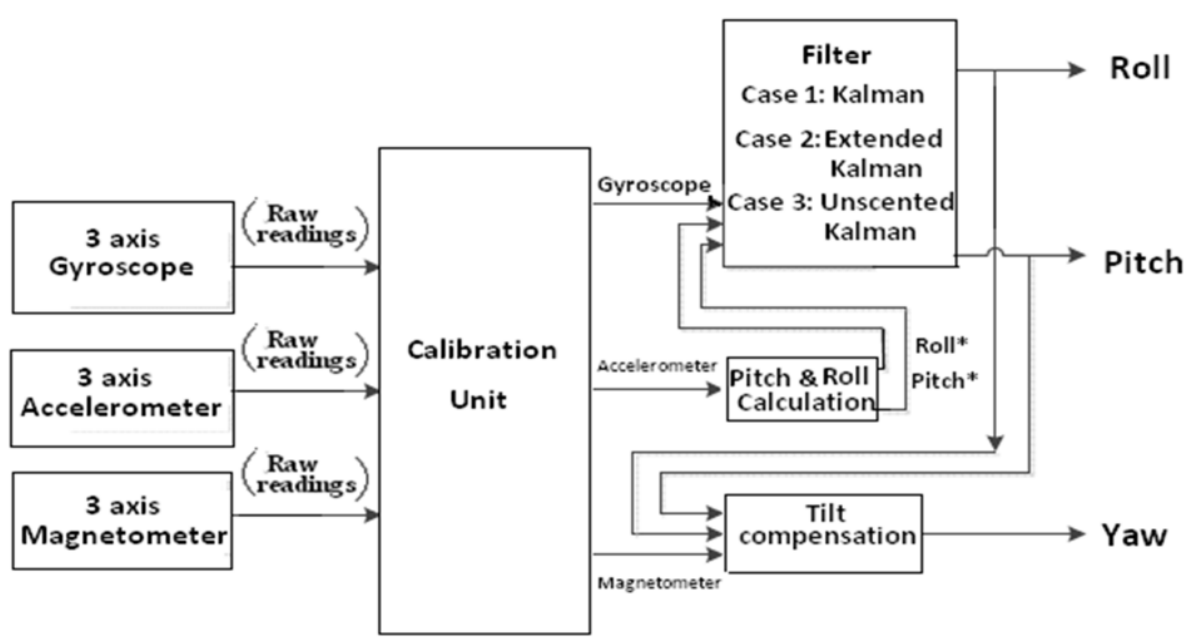

Figure 6. Sensor Fusion Model for the OktoKopter

The first step is to calibrate the raw data. To calibrate these data, scale and bias must be taken into account. The bias represents how far the center of data is from the zero. The scale means how much larger the range of data from the sensor is than the real meaningful data. The basic guidelines and equations provided by Starlino Electronics project [5] are followed to obtain the calibrated output.

As shown in the Figure 5, the calibrated accelerometer signal is used to obtain roll* and pitch* by utilizing Equations 1 and 2. Roll* and pitch* are noisy calculations and the algorithm combines them with the gyroscope signal through a Kalman filter to acquire clean and not-drifting roll and pitch angles. On other hand, a tilt compensation unit is implemented, which uses a magnetometer signal in combination with roll and pitch to calculate the yaw rotation. At the first instance, we select Kalman filter algorithm (case1), later we apply extended Kalman filter (case 2) and unscented Kalman filter algorithm (case3).

\section{State Estimation and Filter Algorithms}

State estimation concerns the problem of estimating the probability density function (PDF) for the state of a process, which is not directly observable. This involves both, predicting the next state based on the current, and updating/correcting this prediction based on the obtained noisy measurements.

Since many years, Kalman filter $(\mathrm{KF})$ remained a popular estimation algorithm in many control applications. As many new applications were developed, accurately estimating the state of systems became extremely important for fault detection and control applications. As a result, many new algorithms were developed based on basic Kalman dynamics [10]. In the following section we discuss Kalman filter and the other two variants, namely extended Kalman and unscented Kalman filter in brief. 


\subsection{Kalman Filter}

The Kalman filter has been the subject of extensive research and application, especially in the autonomous area. The Kalman filter is a set of mathematical equations that gives an efficient computation in estimating the state of a process and also a powerful tool to estimate the past, present, and even future states. The filter is used to minimize the mean of the squared error. The filter has expanded its application to position and orientation tracking, image processing filter and navigation system [11].

Based on [10], the filter estimates the process state at some time and then obtains feedback in the form of measurement. The Kalman filter is divided into two main groups of equations, time update equations and measurement update equations. In time update equations, it computes the current state by projecting forward in time and error covariance estimates to obtain the a priori estimates for the next time step. In measurement update equation, it incorporates with a new measurement into the a-priori estimate to obtain an improved a posteriori estimate. The time update equation is thought as predictor equation, while the measurement update equation is thought as corrector equation. The basic Kalman filter cycle can be described as shown as Figure 7. The symbols and their respective definitions are given in Table 2.

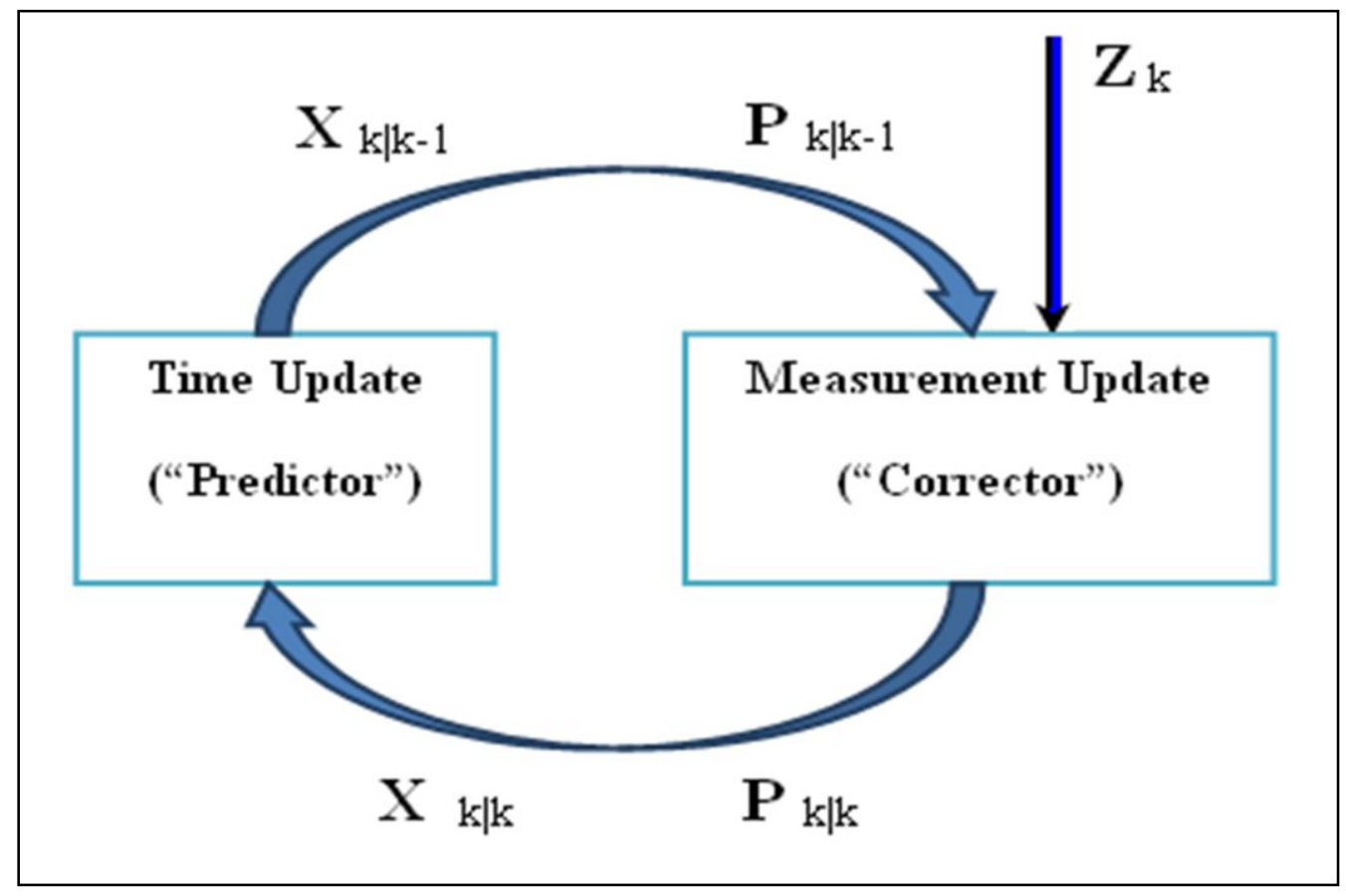

Figure 7. Kalman Filter Cycle 
Table 2. Basic Kalman Filtering Notations

\begin{tabular}{|c|c|}
\hline Symbols & Definitions \\
\hline $\mathrm{F}$ & $\begin{array}{l}\text { Dynamic coefficient matrix (state matrix) of a continuous linear differential equation } \\
\text { defining a dynamic system. }\end{array}$ \\
\hline $\mathrm{G}$ & Coupling matrix between random process noise and the state of a dynamic system \\
\hline $\mathrm{H}$ & $\begin{array}{l}\text { Measurement sensitivity matrix defining the linear relationship between the state of } \\
\text { the dynamic system and measurements that can be made, (also known as a coefficient } \\
\text { matrix. }\end{array}$ \\
\hline $\mathrm{K}$ & Kalman gain matrix \\
\hline $\mathrm{P}$ & Covariance matrix of state estimation uncertainty \\
\hline Q & $\begin{array}{l}\text { Covariance matrix of process noise in the system state dynamics also called the process } \\
\text { noise covariance. }\end{array}$ \\
\hline $\mathrm{R}$ & $\begin{array}{l}\text { Covariance matrix of observational (measurement) uncertainty also called the } \\
\text { measurement noise covariance }\end{array}$ \\
\hline$x$ & State vector \\
\hline$y$ & Vector (or scalar) of measured values. \\
\hline$\Phi$ & State transition matrix of a discrete dynamic system \\
\hline$X_{k}(i)$ & $\begin{array}{l}\text { The } i \text {-th component of the vector } \mathrm{x} \text {, or the } i \text {-th element of the sequence. The sub-index } \\
\mathrm{k} \text { refers to the sequence of propagation as it occurs in the filtering process. i.e. } k+1 \text { can } \\
\text { be referred to as the "update" term that is determined from the same term calculated } \\
\text { previously. } X_{\mathrm{k}+1}(i)=X_{\mathrm{k}}(i)+\text { noise }\end{array}$ \\
\hline$\hat{x}$ & An estimate of the value of $x$. \\
\hline$\hat{x}_{k}^{-}$ & $\begin{array}{l}\text { A priori estimate of the } X_{k} \text {, conditioned on all prior measurements except the one at } \\
\text { time } t_{k} \text {. }\end{array}$ \\
\hline$\hat{x}_{k}^{+}$ & A posteriori estimate of the $x$, conditioned on all available measurements at time $t_{k}$. \\
\hline$\tilde{y}$ & A measurement of some quantity we can estimate to the state vector from. \\
\hline$\dot{x}$ & De \\
\hline
\end{tabular}

In the time update equations, equations are used to compute the state and error covariance are projected ahead. The measurement update equation are used to compute the Kalman gain and error covariance and used to update estimate with current measurement. These update and measurement equations for the Kalman filter are as follows [10]:

Time Update:

(1)Project the state ahead

$$
\hat{x}_{k}^{-}=A \hat{x}_{k-1}+B u_{k-1} \quad \text { Equation (3) }
$$

(2) Project the error covariance ahead

$$
P_{k}^{-}=A P_{k-1} A^{T}+Q \quad \text { Equation (4) }
$$

Measurement Update:

(1) Compute the Kalman gain 


$$
K_{k}=P_{k}^{-} H^{T}\left(H P_{k}^{-} H^{T}+R\right)^{-1}
$$

(2) Update estimate with measurement $z_{k}$

$$
\hat{x}_{k}=\hat{x}_{k}^{-}+K_{k}\left(z_{k}-H \hat{x}_{k}^{-}\right)
$$

Equation (6)

(3)Update the error covariance

$$
P_{k}=\left(I-K_{k} H\right) P_{k}^{-}
$$

Equation (7)

\subsection{Extended Kalman Filter}

Kalman filter is an optimal estimator for linear systems, but unfortunately, very few systems in real world are linear. When either the system state dynamics or the observation dynamics is nonlinear, the conditional probability density functions that provide the minimum mean-square estimate are no longer Gaussian. The optimal non-linear filter propagates these non-Gaussian functions and evaluate their mean, which represents a high computational burden. A non-optimal approach to solve the problem, in the frame of linear filters, is the extended Kalman filter (EKF). The EKF implements a Kalman filter for a system dynamics that results from the linearization of the original non-linear filter dynamics around the previous state estimates [12].

Most processes in real life are unfortunately not linear, and therefore needs to be linearized before they can be estimated by means of a Kalman filter. The extended Kalman filter (EKF) solves this problem by calculating the Jacobian (The Jacobian is the matrix of all partial derivatives of a vector) of $f$ and $h$ around the estimated state, which in turn yields a trajectory of the model function centered around this state [13-14].

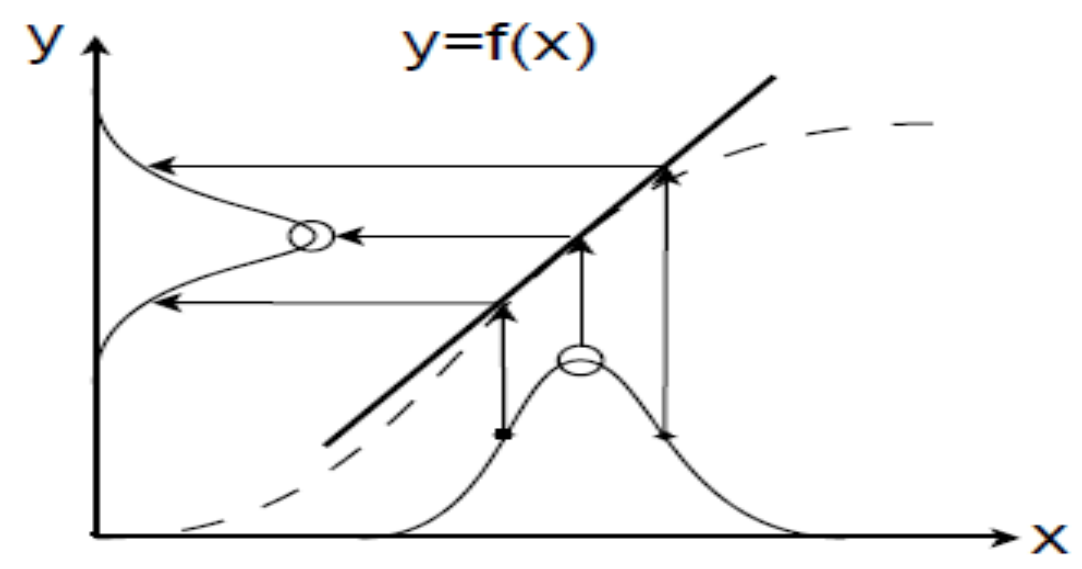

Figure 8. Linearization Graph for Extended Kalman Filter [13]

Figure 8 illustrates how the EKF linearizes a nonlinear function around the mean of a Gaussian distribution, and thereafter propagates the mean and covariance through this linearized model. The equations are as follows [13]:

$$
F_{k}=\left.\frac{\partial f(\mathbf{x}, \mathbf{u}, \mathbf{w})}{\partial \mathbf{x}}\right|_{\hat{\mathbf{x}}_{k \mid k}, \mathbf{u}_{k}, 0} \quad \text { Equation (8) }
$$




$$
H_{k}=\left.\frac{\partial h(\mathbf{x}, \mathbf{v})}{\partial \mathbf{x}}\right|_{\hat{\mathbf{x}}_{k \mid k-1}, 0} \quad \text { Equation (9) }
$$

The extended Kalman filter works almost like a regular Kalman filter, except for $\mathrm{F}$ and $\mathrm{H}$, which vary in time based on the estimated state $X$ (hat). The actual calculations required are: Predict next state, before the measurements are taken. Thus, the resulting equations are as follows [13-14],

$$
\begin{array}{ll}
\hat{\mathbf{x}}_{k \mid k-1}=f\left(\hat{\mathbf{x}}_{k-1 \mid k-1}, \mathbf{u}_{k}, 0\right) & \text { Equation (10) } \\
P_{k \mid k-1}=F_{k} P_{k-1 \mid k-1} F_{k}^{T}+Q_{k} & \text { Equation (11) }
\end{array}
$$

Update state, after measurements are taken:

$$
\begin{array}{ll}
K_{k}=P_{k \mid k-1} H_{k}^{T}\left(H_{k} P_{k \mid k-1} H_{k}^{T}+R_{k}\right)^{-1} & \text { Equation (12) } \\
\hat{\mathbf{x}}_{k \mid k}=\hat{\mathbf{x}}_{k \mid k-1}+K_{k}\left(\mathbf{z}_{k}-h\left(\hat{\mathbf{x}}_{k \mid k-1}, 0\right)\right) & \text { Equation (13) } \\
P_{k \mid k}=\left(I-K_{k} H_{k}\right) P_{k \mid k-1} &
\end{array}
$$

Where $K$ is the Kalman gain matrix, used in the update observer, and $P$ is the covariance matrix for the state estimate, containing information about the accuracy of the estimate.

\subsection{Unscented Kalman Filter}

The problem of propagating Gaussian random variables through a nonlinear function could also be approached using another technique, namely the unscented transform. Instead of linearizing the functions, this transform uses a set of points, and propagates them through the actual nonlinear function, eliminating linearization altogether. The points are chosen such that their mean, covariance, and possibly also higher order moments, match the Gaussian random variable. Mean and covariance can be recalculated from the propagated points, yielding more accurate results compared to ordinary function linearization. The underlying idea is also to approximate the probability distribution instead of the function. Thus, this strategy typically 
does both decrease the computational complexity, while at the same time increasing estimate accuracy, yielding faster, more accurate results [15].

The underlying method of unscented transform was first proposed by Uhlmann et al. in [16] and [17], where they laid out the framework for representing a Gaussian random variable in $\mathrm{N}$ dimensions using $2 \mathrm{~N}+1$ samples, called sigma points. They utilized the matrix square root and covariance definitions to select these points in such a way that they had the same covariance as the Gaussian they approximated. Skewness was avoided by selecting the points in a symmetric way, such that any approximation error would only originate from the fourth and higher moments. Usage of the unscented transform in Kalman filtering was then presented by Julier and Uhlmann in [18], where they introduced the unscented Kalman filter (UKF), which approximates the state estimate using the sigma points. Later, it was analyzed more in depth in [19].

The unscented transform approach also has another advantage that, the noise can be treated in a nonlinear fashion to account for non-Gaussian or non-additive noises. The strategy for doing so involves propagation of noise through the functions by first augmenting the state vector to also include noise sources, a technique first introduced by Julier in [20], and later refined more in depth by Merwe in [21]. Sigma point samples are then selected from the augmented state, $\mathrm{X}^{\mathrm{a}}$, which also includes noise values. The net result is that any nonlinear effects of process and measurement noise are captured with the same accuracy as the rest of the state, which in turn increases accuracy for non-additive noise sources.

The filter starts by augmenting the state vector to $\mathrm{L}$ dimensions, where $\mathrm{L}$ is the sum of dimensions in the original state-vector, model noise and measurement noise. The covariance matrix is similarly augmented to a $\mathrm{L}^{2}$ matrix. Together this forms the augmented state estimate vector $\mathrm{X}^{\mathrm{a}}$ and covariance matrix $\mathrm{P}^{\mathrm{a}}$.

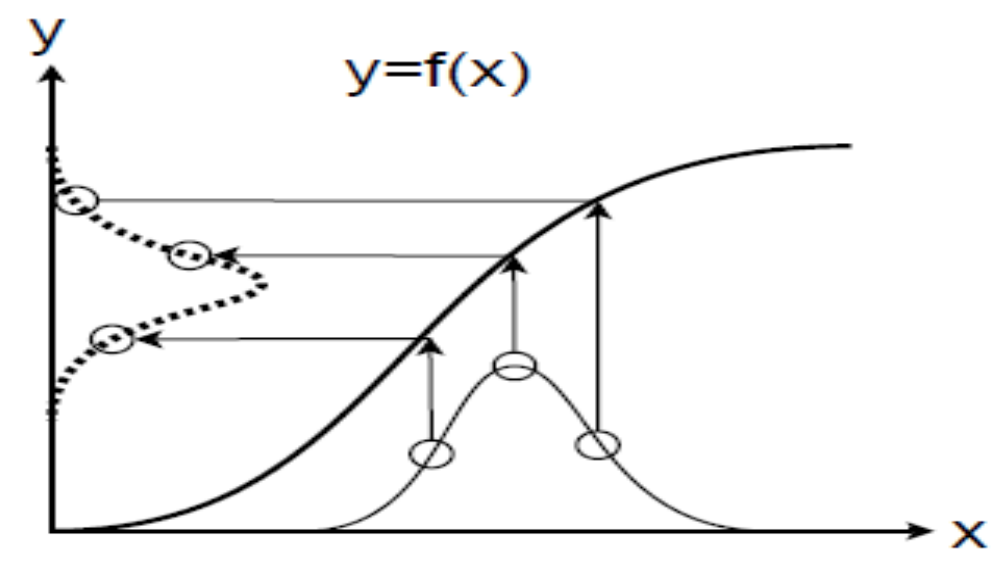

Figure 9. Linearization Graph for Unscented Kalman Filter [13]

Figure 9 illustrates how the unscented Kalman filter propagates sigma-points from a Gaussian distribution through a nonlinear function, and recreates a Gaussian distribution, by calculating the mean and covariance of the results. The equations are as follows [13]:

$$
\mathbf{x}_{k-1}^{a}=\left[\begin{array}{c}
\mathbf{x}_{k-1} \\
0_{w} \\
0_{y}
\end{array}\right]
$$




$$
\begin{aligned}
& P_{k-1}^{a}=E\left\{\left(\mathbf{x}_{k-1}^{a}-\hat{\mathbf{x}}_{k-1}^{a}\right)\left(\mathbf{x}_{k-1}^{a}-\hat{\mathbf{x}}_{k-1}^{a}\right)^{T}\right\} \quad \text { Equation (16) } \\
& =\left[\begin{array}{ccc}
P_{k-1} & 0 & 0 \\
0 & Q_{k-1} & 0 \\
0 & 0 & R_{k-1}
\end{array}\right]
\end{aligned}
$$

Equation (17)

The next step consists of creating $2 \mathrm{~L}+1$ sigma-points in such a way that they together capture the full mean and covariance of the augmented state. The $\chi^{\mathrm{a}}$ matrix is chosen to contain these points, and its columns are calculated as follows:

$$
\begin{aligned}
& \chi_{0, k-1}^{a}=\mathbf{x}_{k-1}^{a} \quad i=0 \\
& \chi_{i, k-1}^{a}=\mathbf{x}_{k-1}^{a}+\left(\alpha \sqrt{L P_{k-1}^{a}}\right)_{i}, \quad i=1 \ldots, L \quad \text { Equation (19) } \\
& \chi_{i, k-1}^{a}=\mathbf{x}_{k-1}^{a}-\left(\alpha \sqrt{L P_{k-1}^{a}}\right)_{i-L}, \quad i=L+1 \ldots, 2 L \quad \text { Equation (20) }
\end{aligned}
$$

Where subscript $i$ means the $\mathrm{i}$-th column of the square root of the covariance matrix. (The square root of a symmetric matrix is typically calculated by means of a lower triangular Cholesky decomposition. The square root $\mathrm{A}$ of matrix $\mathrm{P}$ is then on the form $\mathrm{P}=\mathrm{AA}^{\mathrm{T}}$ ).

The $\alpha$ parameter, in the interval $0<\alpha \leq 1$, determines sigma-point spread. This parameter is typically quite low, often around 0.001 , to avoid non-local effects.

The resulting $\chi_{k-1}^{\mathrm{a}}$ matrix can now be decomposed vertically into the $\chi_{k-1}^{x}$ rows, which contain the state; the $\chi_{k-1}^{w}$ rows, which contains sampled process noise and the $\chi_{k-1}^{v}$ rows, which contains sampled measurement noise.

Each sigma-point is also assigned a weight. These weight are derived by comparing the moments of the sigma-points with a Taylor series expansion of the models while assuming a Gaussian distribution, as derived in [22]. The resulting weights for mean (m) and covariance (c) estimates then becomes:

$$
\begin{array}{lll}
w_{0}^{(m)}=1-\frac{1}{\alpha^{2}} & i=0 & \text { Equation (21) } \\
w_{0}^{(c)}=4-\frac{1}{\alpha^{2}}-\alpha^{2} & i=0 & \text { Equation (22) } \\
w_{i}^{(m)}=w_{i}^{(c)}=\frac{1}{2 \alpha^{2} L} & i=1 \ldots, 2 L & \text { Equation (23) }
\end{array}
$$

The filter then predicts next state by propagating the sigma-points through the state and measurement models, and then calculating weighted averages and covariance matrices of the results: 


$$
\begin{gathered}
\chi_{k \mid k-1}^{x}=f\left(\chi_{k-1}^{x}, \mathbf{u}_{k}, \chi_{k-1}^{w}\right) \\
-1=\sum_{i=0}^{2 L} w_{i}^{(m)} \chi_{k \mid k-1}^{x} \\
P_{k \mid k-1}=\sum_{i=0}^{2 L} w_{i}^{(c)}\left[\chi_{k \mid k-1}^{x}-\hat{\mathbf{x}}_{k \mid k-1}\right]\left[\chi_{k \mid k-1}^{x}-\hat{\mathbf{x}}_{k \mid k-1}\right]^{T} \\
Z_{k \mid k-1}=h\left(\chi_{k \mid k-1}^{x}, \chi_{k-1}^{v}\right) \\
\hat{\mathbf{z}}_{k \mid k-1}=\sum_{i=0}^{2 L} w_{i}^{(m)} Z_{i, k \mid k-1}
\end{gathered}
$$

Equation (27)

The predictions are then updated with new measurements by first calculating the measurement covariance and state-measurement cross correlation matrices, which are then used to determine the Kalman gain:

$$
\begin{aligned}
& P_{z z}=\sum_{i=0}^{2 L} w_{i}^{(c)}\left[Z_{i, k \mid k-1}-\hat{\mathbf{z}}_{k \mid k-1}\right]\left[Z_{i, k \mid k-1}-\hat{\mathbf{z}}_{k \mid k-1}\right]^{T} \\
& P_{x z}=\sum_{i=0}^{2 L} w_{i}^{(c)}\left[\chi_{i, k \mid k-1}^{x}-\hat{\mathbf{x}}_{k \mid k-1}\right]\left[Z_{i, k \mid k-1}-\hat{\mathbf{z}}_{k \mid k-1}\right]^{T} \\
& K_{k}=P_{x z} P_{z z}^{-1} \\
& \hat{\mathbf{x}}_{k \mid k}=\hat{\mathbf{x}}_{k \mid k-1}+K_{k}\left(\mathbf{z}_{k}-\hat{\mathbf{z}}_{k \mid k-1}\right) \\
& P_{k \mid k}=P_{k \mid k-1}-K_{k} P_{y y} K_{k}^{T}
\end{aligned}
$$

As we are implementing the sensor fusion model described in Figure 6, we consider case by case, thus the change in the equations for each case is properly interpreted using Matlab programming, which is obviously reflected in the final results.

\section{Results and Discussions}

Matlab simulations are carried out for all the three algorithms. The obvious change in the raw output and the fused filtered output from all the three sensors are depicted in Figures 10 15. 


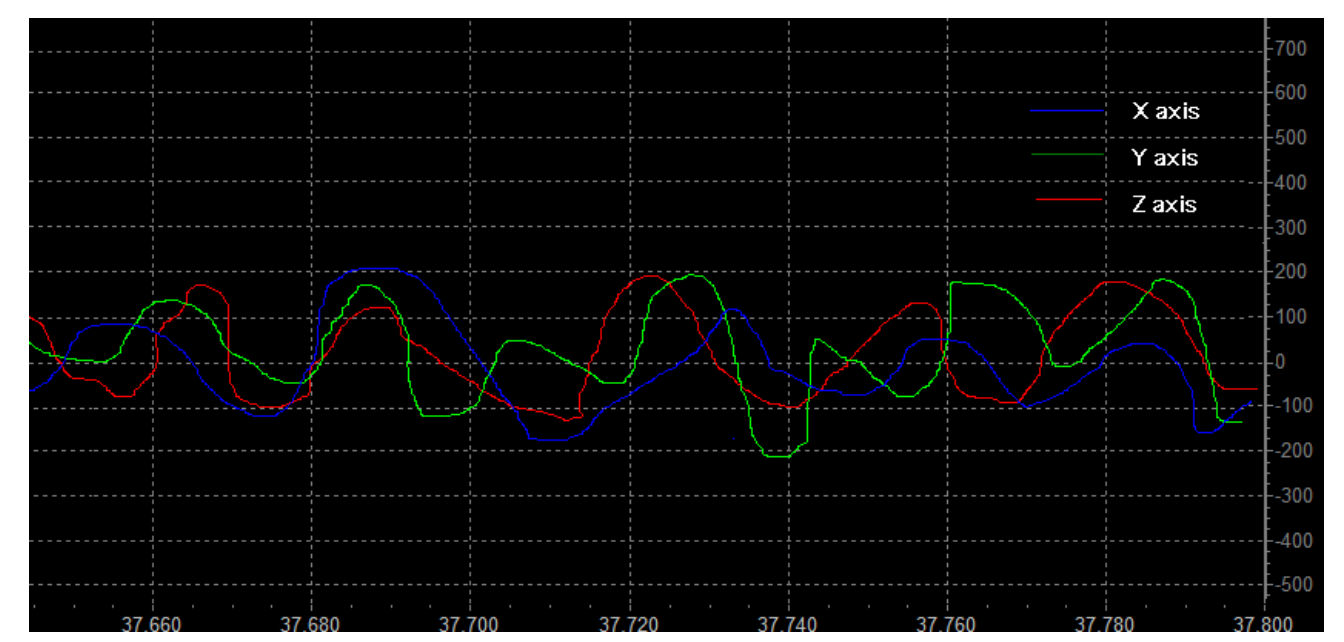

Figure 10. Gyroscope Raw Output

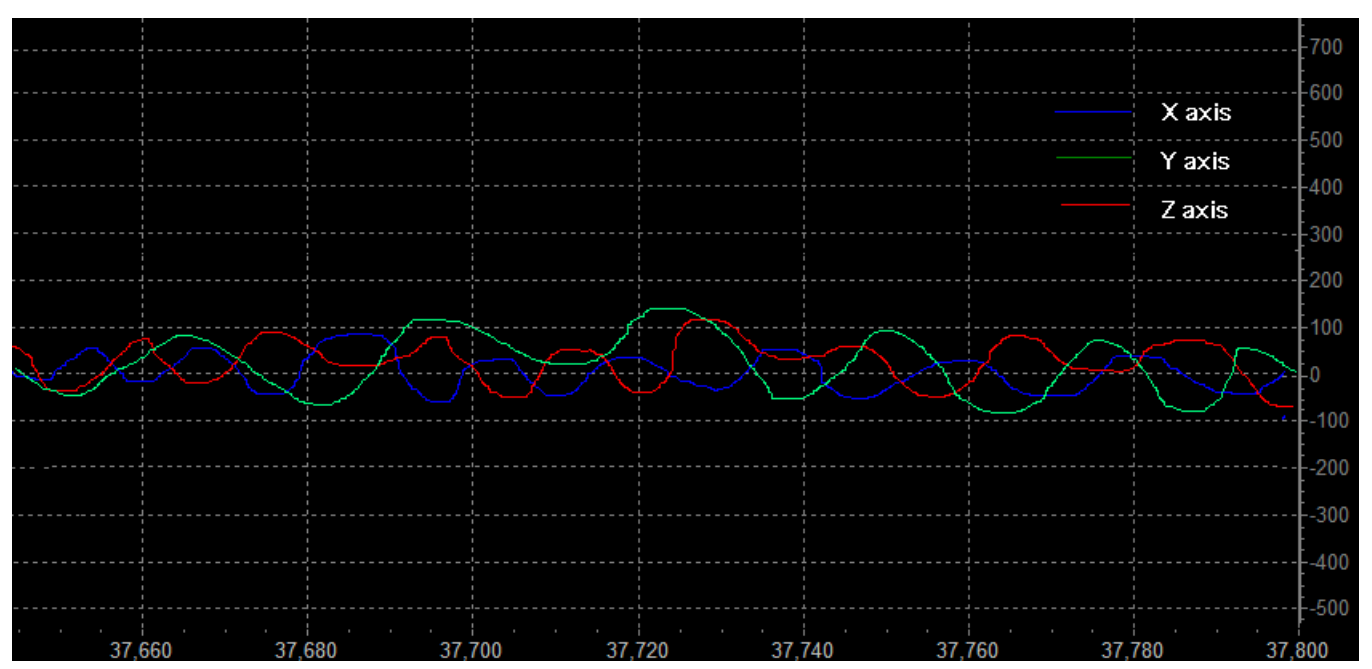

Figure 11. Gyroscope Filtered Output

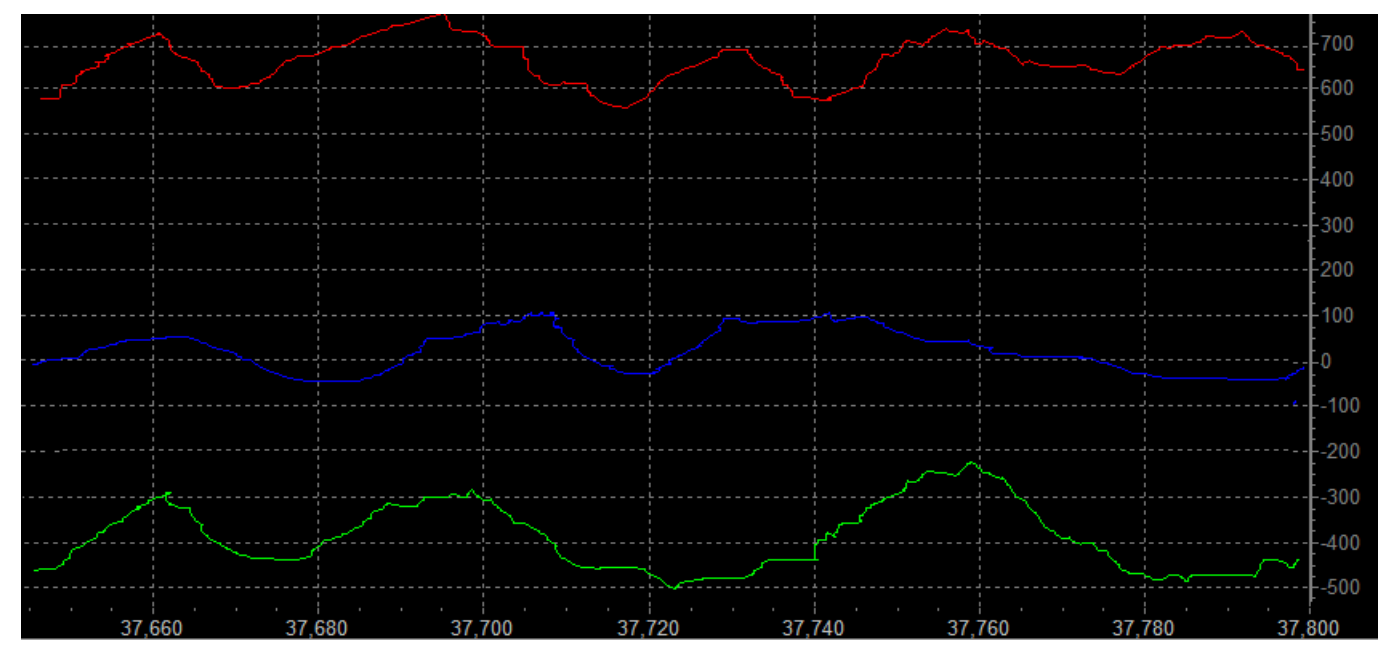

Figure 12. Accelerometer Raw Output 


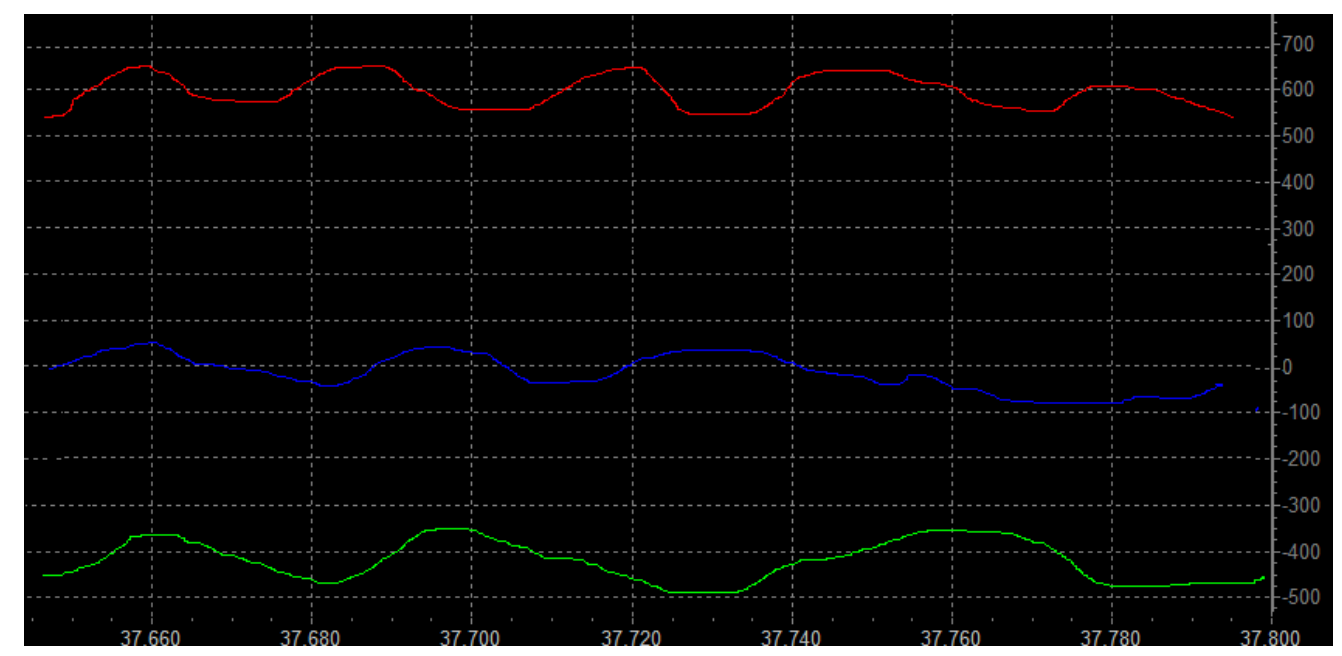

Figure 13. Accelerometer Filtered Output

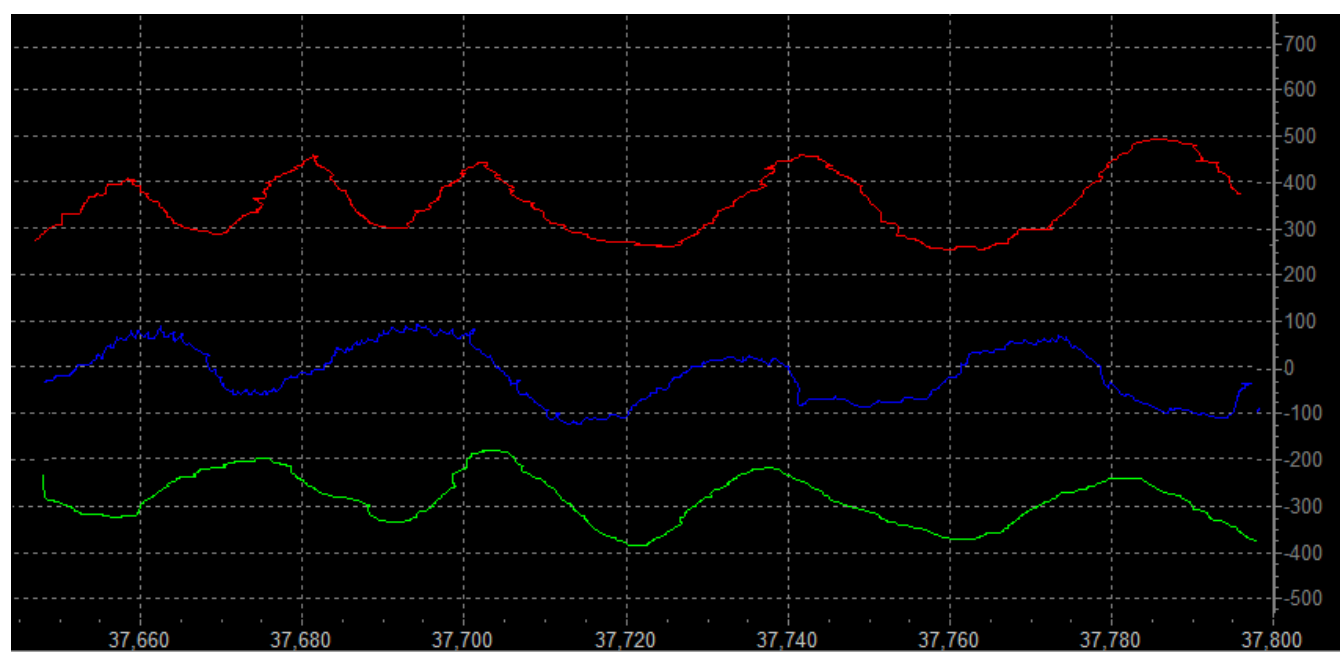

Figure 14. Magnetometer Raw Output

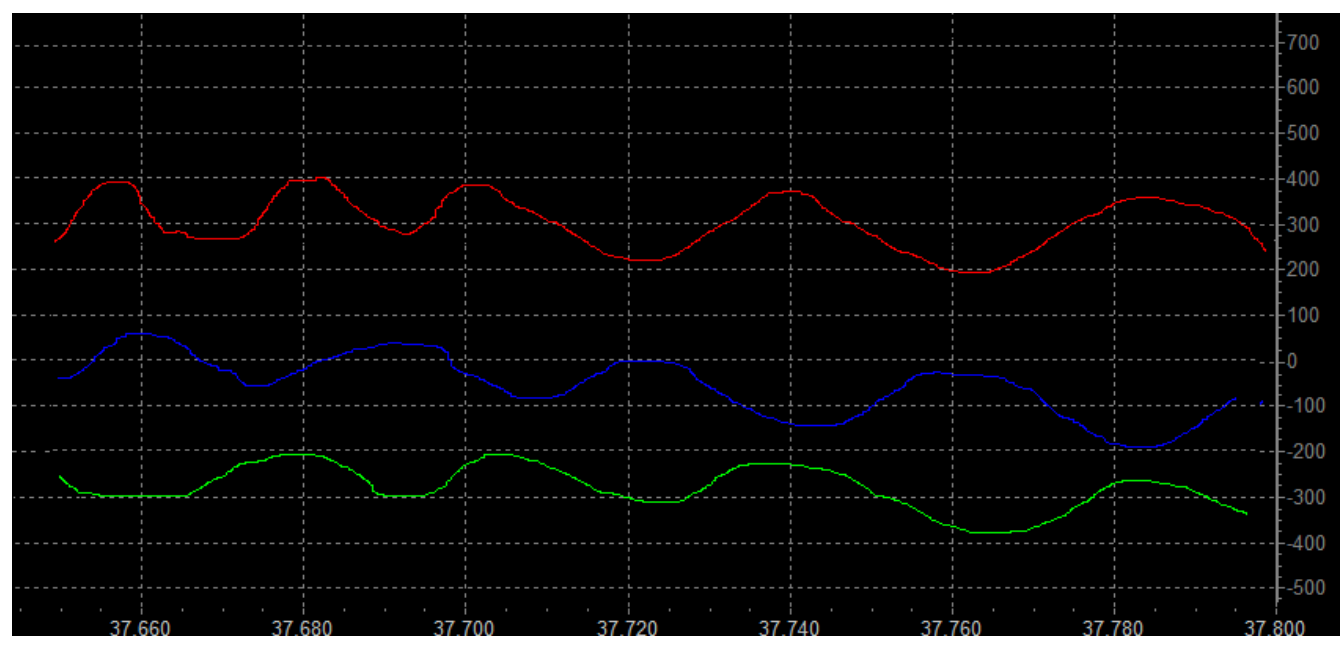

Figure 15. Magnetometer Filtered Output 
In order to compare all the three algorithms, we have considered estimation error as a point of reference. Although, drastic differences were not visible, but unscented Kalman filter has outperformed extended Kalman and Kalman filter. As shown in Figure 16, the estimation error shown for UKF (blue color) is less than EKF (red color), which is less than Kalman filter (green color).

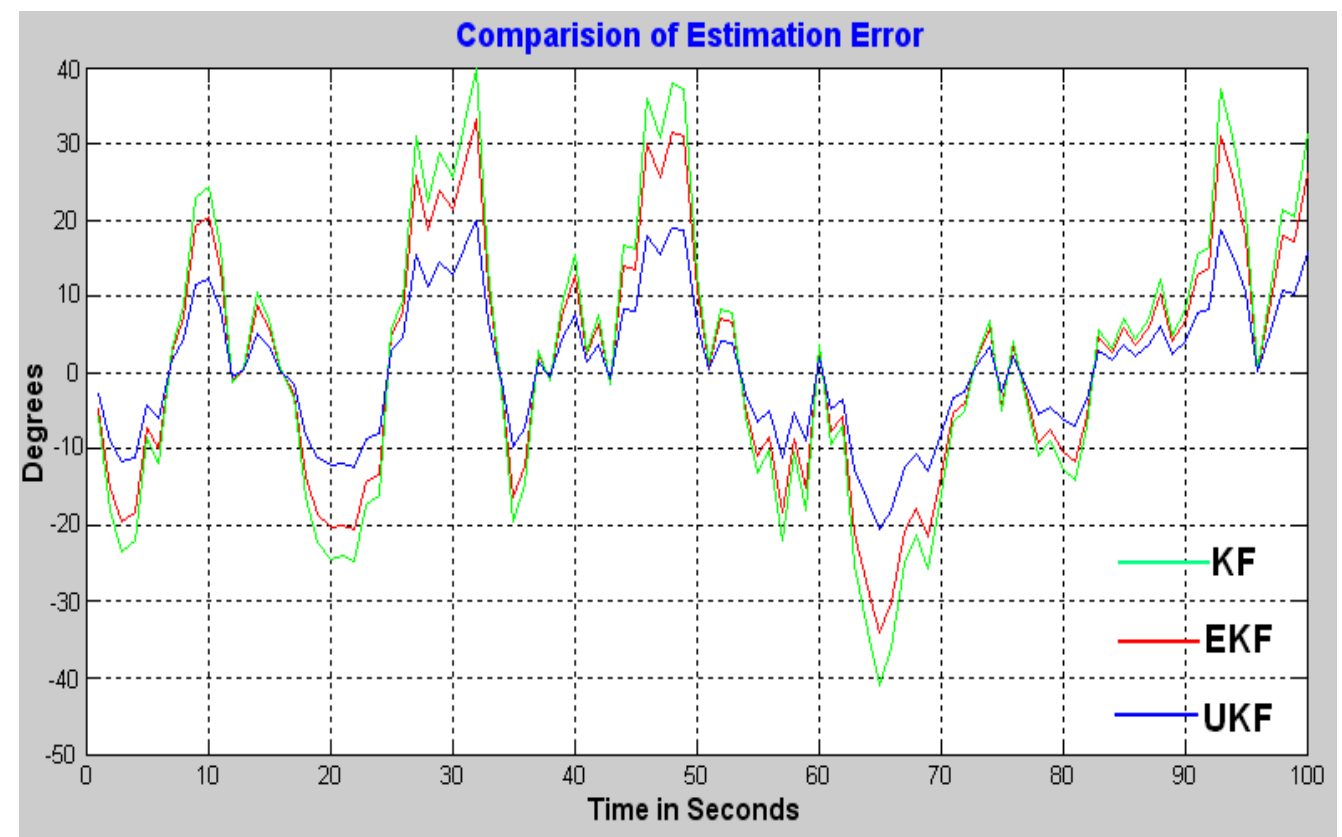

Figure 16. Comparison of Estimation Errors of Three Filters

Thus from the theoretical perspective also it is comprehensible that the state estimation approach adopted in UKF is better than EKF and KF. Consequently, the experimental results shown in Figure 16 are corroborated.

\section{Conclusion}

The development of better estimator algorithms for nonlinear systems has attracted a great deal of interest in the aerospace engineering system. In addition, with the introduction of OktoKopter and related basics, the paper is focused on exploring the best state estimation approach. We have proposed a sensor fusion model that primarily facilitates fusion of accelerometer and gyroscope readings in order to provide better inferences, secondarily it provides an option for the selection of estimation algorithm. Simulation results reveal that the estimation error in case of unscented Kalman filter is least as compared to extended Kalman filter and Kalman filter, thus make it a better choice for the estimator algorithm.

\section{References}

[1] http://www.cedec.usm.my/, (2014) May.

[2] http://www.mikrokopter.de/ucwiki/Manual, (2014) May.

[3] P. J. Costa, "Adaptive Model Architecture and Extended Kalman - Bucy Filters", IEEE Transactions on Aerospace and Electronic Systems, vol. 30, no. 2, (1994), pp. 525-533.

[4] G. Prasad, G.W. Irwin, E. Swidenbank, and B.W. Hogg, "Plant-wide Predictive Control for a Thermal Power Plant based on a Physical Plant Model”, IEE Proceedings on Control Theory Application, vol. 147, no. 5, (2000), pp. 523-537. 
[5] http://www.starlino.com/imu_guide.html, (2014) May.

[6] O. Sidek and S.A.Quadri, "A Review of Data Fusion Models and Systems", International Journal of Image and Data Fusion, vol. 3, no. 1, (2012), pp.3-21.

[7] http://home.comcast.net/ michael.p.thompson/kalman/kalman_test2.c, (2014) May.

[8] S.A.Quadri and O. Sidek, "Factors Affecting Data Fusion Performance in an Inertial Measurement Unit", Journal of Control Engineering and Technology (JCET), vol. 3, no. 3 (2013), pp. 107-110.

[9] S.A.Quadri and O. Sidek, "Multisensor Data Fusion Algorithm using Factor Analysis Method", International Journal of Advanced Science and Technology, vol. 55, (2013), pp.43-52.

[10] G. Welch and G. Bishop, "An Introduction to the Kalman Filter," Department of Computer Science, University of North Carolina, Chapel Hill, (2006), pp. 1-16. online available: http://www.cs.unc.edu/ welch/kalman/kalmanIntro.html, (2014) May.

[11] C. Kownacki, "Optimization Approach to Adapt Kalman Filters for the Real-time Application of Accelerometer and Gyroscope Signals Filtering”, Digital Signal Processing, vol. 21, no.1, (2011), pp. 131140.

[12] G.G. Rigatos, "Nonlinear Kalman Filters and Particle Filters for Integrated Navigation of Unmanned Aerial Vehicles", Robotics and Autonomous Systems, vol. 60, no. 7, (2012), pp. 978-995

[13] O.X. Diaz, "Analysis and Comparison of Extended and Unscented Kalman Filtering Methods for Spacecraft Attitude Determination”, Master Thesis, Naval Postgraduate School, The University of Texas, Austin, (2004). online available:http://calhoun.nps.edu/public/bitstream/handle/10945/5010/10Dec_Diaz.pdf?sequence=1 (2014) May.

[14] F. Orderud, "Comparison of Kalman Filter Estimation Approaches for State Space Models with Nonlinear Measurements", Sem Sælands vei, vol. 7491, (2006), pp. 7-9.

[15] J. L. Crassidis and J. L. Junkins, "Optimal Estimation of Dynamic Systems", Chapman \& Hall, New York, 2nd edition, (2004), pp.320- 340.

[16] B. Quine, J. Uhlmann, and H. Durrant-Whyte, "Implicit Jacobian for Linearised State Estimation in Nonlinear Systems", Proceedings of the American Control Conference, (1995); Seattle, WA.

[17] S. Julier, J. Uhlmann, and Durrant-Whyte, H, "A New Approach for Filtering Nonlinear Systems", Proceedings of the American Control Conference, (1995); Seattle, WA.

[18] S. J. Julier and J. K. Uhlmann, "New Extension of the Kalman Filter to Nonlinear Systems", Proceedings of International Symposium on Aerospace/Defense Sensing, Simulations and Controls, (1997); Orlando, FL.

[19] E. Wan and R. Merwe, "The Unscented Kalman Filter for Nonlinear Estimation", Proceedings of the IEEE Symposium on Adaptive Systems for Signal Processing, Communications, and Control, (2000); Alberta, Canada.

[20] S. Julier and J. Uhlmann, "A General Method for Approximating Nonlinear Transformations of Probability Distributions", University of Oxford, (1996).

online available: http://www.smpp.northwestern.edu/savedLiterature/JulierUhlmannUnscented.pdf (2014) May.

[21] R. Merwe and E. Wan, "Sigma-Point Kalman Filters for Integrated Navigation", Proceedings of the 60th Annual Meeting of The Institute of Navigation, (2004); Dayton, Ohio.

[22] S. J. Julier, and J.K Uhlmann, "Unscented Filtering and Nonlinear Estimation", Proceedings of the IEEE, vol. 92 , no. 3 , (2004).

\section{Authors}

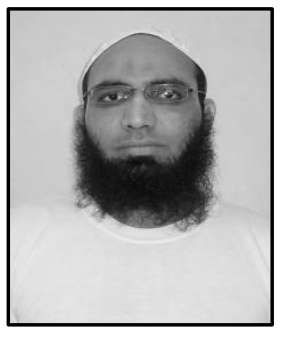

S. A. Quadri graduated as Bachelor of Engineering in Electronics \& Communication from Karnataka University Dharwad. He completed his M.Tech (Computer Science \& Engineering) from VTU Belgaum. He served as Assistant professor in various Engineering colleges. Before joining as Research associate in CEDEC he served 3 years as HOD of Computer Science \& Engineering department in SIET College, affiliated to VTU, approved by AICTE Delhi, India. His research domain includes multisensor data fusion \& neural networks. 


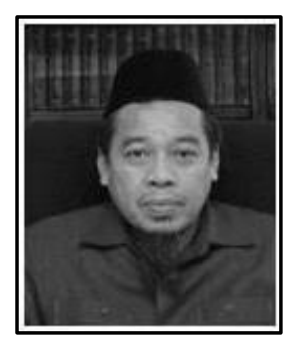

Professor Othman bin Sidek an esteemed Malaysian scientist holds a $\mathrm{PhD}$ (Info. Sys. Eng.) from Bradford University, UK. He has served Universiti Sains Malaysia since 1984 as a lecturer, researcher, \& administrator. Prior to joining USM, he was a trainee engineer with Multinational Companies \& continued to take very active role in both academic \& university-industry research collaborations. Professor is also the Founder of the Collaborative Micro-electronic Design Excellence Centre (CEDEC), an approved centre at USM by the Malaysian Ministry of Finance in 2005 out of his own initiative.

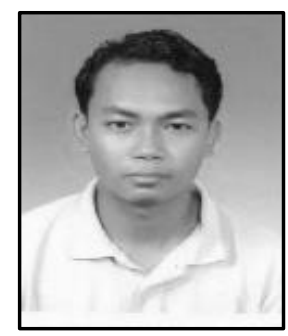

Azizul bin Abdullah graduated from USM with Aerospace Engineering Degree \& Masters in the year of 2008 \& 2012 respectively. Currently doing his research in 'Development of wide secured wireless sensor network (WSN) with unmanned aerial vehicle (UAV) deployment for the monitoring of ecology \& global warming studies towards environment sustainability' and are the one who analysis designs of Agrotech 2020 UAV. 
International Journal of $u$ - and e- Service, Science and Technology Vol.7, No.3 (2014) 